\title{
BMJ Open Values and preferences towards medical cannabis among people living with chronic pain: a mixed-methods systematic review
}

\author{
Linan Zeng (D) , ${ }^{1,2}$ Lyubov Lytvyn, ${ }^{2}$ Xiaoqin Wang, ${ }^{3}$ Natasha Kithulegoda (D) ,4,5 \\ Silvana Agterberg, ${ }^{6}$ Yaad Shergill, ${ }^{2}$ Meisam Abdar Esfahani, ${ }^{2}$ Anja Fog Heen, ${ }^{7}$ \\ Thomas Agoritsas (D) , ${ }^{2,8}$ Gordon H Guyatt, ${ }^{2}$ Jason W Busse (D) ${ }^{2,9,10,11,12}$
}

To cite: Zeng L, Lytvyn L, Wang $X$, et al. Values and preferences towards medical cannabis among people living with chronic pain: a mixed-methods systematic review. BMJ Open 2021;11:e050831. doi:10.1136/ bmjopen-2021-050831

- Prepublication history and additional online supplemental material for this paper are available online. To view these files, please visit the journal online (http://dx.doi.org/10. 1136/bmjopen-2021-050831)

Received 08 March 2021 Accepted 24 May 2021

Check for updates

(C) Author(s) (or their employer(s)) 2021. Re-use permitted under CC BY-NC. No commercial re-use. See rights and permissions. Published by BMJ.

For numbered affiliations see end of article.

Correspondence to Professor Jason W Busse; bussejw@mcmaster.ca

\section{ABSTRACT}

Objective To explore values and preferences towards medical cannabis among people living with chronic pain.

Design Mixed-methods systematic review.

Data sources We searched MEDLINE, EMBASE and

PsycINF0 from inception to 17 March 2020.

Study selection Pairs of reviewers independently screened search results and included quantitative, qualitative and mixed-methods studies reporting values and preferences towards medical cannabis among people living with chronic pain.

Review methods We analysed data using meta-narrative synthesis (quantitative findings were qualitised) and tabulated review findings according to identified themes. We used the Grading of Recommendations Assessment, Development and Evaluation approach to assess certainty of evidence.

Results 0 1838 initial records, 15 studies proved eligible for review. High to moderate certainty evidence showed that patient's use of medical cannabis for chronic pain was influenced by both positive (eg, support from friends and family) and negative social factors (eg, stigma surrounding cannabis use). Most patients using medical cannabis favoured products with balanced ratios of tetrahydrocannabinol (THC) and cannabidiol (CBD), or high levels of CBD, but not high THC preparations. Many valued the effectiveness of medical cannabis for symptom management even when experiencing adverse events related to concentration, memory or fatigue. Reducing use of prescription medication was a motivating factor for use of medical cannabis, and concerns regarding addiction, losing control or acting strangely were disincentives. Outof-pocket costs were a barrier, whereas legalisation of medical cannabis improved access and incentivised use. Low to very low certainty evidence suggested highly variable values towards medical cannabis among people living with chronic pain. Individuals with pain related to life-limiting disease were more willing to use medical cannabis, and preferred oral over inhaled administration. Conclusions Our findings highlight factors that clinicians should consider when discussing medical cannabis. The variability of patients' values and preferences emphasise the need for shared decision making when considering medical cannabis for chronic pain.
Strengths and limitations of this study

- Consideration of complementary bodies of evidence (qualitative, quantitative and mixed methods) and use of the Grading of Recommendations Assessment, Development and Evaluation approach to assess the certainty of evidence provide greater confidence in the interpretation of results.

- Most eligible studies are from high-income countries, reflecting values and preferences of patients living in better healthcare service systems with health insurance coverage. The generalisability of our findings to other populations is uncertain.

- Studies eligible for this review failed to consistently report participants' socioeconomic status, educational level and religious beliefs, limiting exploration of the impact of these characteristics on values and preferences towards medical cannabis for chronic pain.

\section{INTRODUCTION}

Chronic pain is the major cause of non-fatal disease burden worldwide, ${ }^{1}$ and is estimated to affect one in five adults in the general global population $^{2}$ and one in three in low-income and middle-income countries. ${ }^{3}$ Opioids are commonly prescribed for chronic pain; however, increasing awareness of modest benefits and risks of addiction, overdose and death have generated interest for alternative management strategies. Medical cannabis, whose two most studied active ingredients are delta-9tetrahydrocannabinol (THC) and cannabidiol (CBD), is one such therapeutic alternative. ${ }^{4}$ Moreover, the legalisation of medical cannabis among more than 30 countries $^{5}$ has increased access for people living with chronic pain who are considering this option. Accordingly, physicians are increasingly faced with questions from patients about the potential role of medical cannabis in managing their pain. ${ }^{6}$ 
Physicians who seek guidance from current clinical practice guidelines regarding medical cannabis for chronic pain will find recommendations to be inconsistent. As examples, the UK's National Institute for Health and Care Excellence (NICE) recommends against prescribing cannabis-related products for chronic pain, citing its high cost and inadequate supporting evidence. ${ }^{7}$ The American Academy of Neurology recommends an oral cannabis extract containing both THC and CBD as having the highest level of empirical support as a treatment for chronic pain associated with multiple sclerosis. ${ }^{8}$ These guidelines, and others, have neglected to systematically identify and incorporate target patients' values and preferences, which may affect their findings.

Understanding patients' values and preferences, defined as patient-important desirable and undesirable consequences weighed when making a recommendation, ${ }^{9}$ can improve the trustworthiness of recommendations. Therefore, we conducted a systematic review investigating values and preferences towards the use of medical cannabis among people living with chronic pain. This systematic review is part of the BMJ Rapid Recommendations project, a collaborative effort from the MAGIC Evidence Ecosystem Foundation (www.magicevidence. org) and the British Medical Journal. This systematic review informed a parallel guideline published on bmj. com and MAGICapp. ${ }^{10}$

\section{METHODS}

We registered our study protocolon the Open Science Framework (https://osf.io/5d72w) and adhered to the Preferred Reporting Items for Systematic reviews and Meta-Analyses statement.

\section{Data source and searches}

We searched MEDLINE, EMBASE and PsycINFO from inception to 17 March 2020, using a combination of search filters for retrieving studies on values and preferences towards cannabis use among people living with chronic pain (online supplemental appendix 1). ${ }^{11} \mathrm{We}$ reviewed reference lists of all included studies and relevant reviews to identify additional eligible studies.

\section{Study selection}

We included quantitative, qualitative (including survey research that only reported qualitative findings) and mixed-methods studies that reported values and preferences of people living with chronic cancer or non-cancer pain, or their carers, on: (1) relative values or importance of outcomes related to medical cannabis use (eg, improvements in pain and function, side effects) for chronic pain (defined as pain lasting 3 months or longer); (2) formulation of medical cannabis (eg, administration routes, ingestion methods, ratios of THC to CBD) or (3) factors that influence the decision to use medical cannabis. If studies enrolled both acute and chronic pain patients, we considered them eligible if they reported outcomes of chronic pain patients separate from others, or if at least $80 \%$ of patients were affected by chronic pain.

We excluded studies that: (1) did not elicit data from patients or carers directly (eg, data elicited from health providers; information from databases of health records); (2) only reported health state values or quality of life of people living with chronic pain, not related to use of medical cannabis; (3) only reported correlation analyses of associations among demographic variables, other patient characteristics and medical cannabis use for chronic pain; (4) case studies with less than 10 patients; (5) studies published in languages other than English; or (6) abstracts and literature reviews.

Before beginning each phase of the review process, we conducted calibration exercises in which reviewers assessed the same two articles and discussed any disagreements, leading to clarification and a common understanding of criteria and process. After calibration, six paired reviewers (LZ and XW, NK and SA, YS and MAE) independently screened titles and abstracts of all retrieved references, and the full text of articles deemed potentially eligible. We resolved disagreements by discussion or consultation with an adjudicator (LL).

\section{Data collection and risk of bias assessment}

Three pairs of reviewers (LZ and XW, NK and SA, YS and $\mathrm{MAE}$ ) extracted data from eligible studies, independently and in duplicate, for research questions, population characteristics, design and methods of data collection, risk of bias or methodological limitations and main findings (online supplemental appendix 2). For main findings, we selected two eligible articles per study design, identified key themes addressed in the studies, and then coded the themes as different categories for main findings in the data abstraction form (online supplemental appendix 2). ${ }^{12}$ We resolved disagreements through discussion to reach consensus, or in consultation with an adjudicator (LL).

For quantitative studies, we used Grading of Recommendations Assessment, Development and Evaluation (GRADE) guidance for studies of values and preferences to assess risk of bias of individual studies (online supplemental appendix 3$) \cdot{ }^{13}$ For qualitative studies, we used the Critical Appraisal Skills Programme checklist to assess methodological reporting quality of individual studies (online supplemental appendix 4$).{ }^{14}$

\section{Data synthesis and analysis}

Using an iterative process, we compared themes of the categories identified across all studies and developed analytical themes. ${ }^{12}$ We applied critical meta-narrative synthesis, a modified form of critical interpretive synthesis, to transform quantitative into qualitative data using systematic profiles and critical questions that are asked to further extract narratives from the data. ${ }^{15} 16$ To facilitate this transformation, we applied four types of profiles to transform the extracted quantitative data that had the potential to be qualitised, or converted into 
Table 1 Critical meta-narrative synthesis: from quantitative data to narratives

\begin{tabular}{|c|c|c|c|}
\hline \multicolumn{3}{|c|}{ Systematic profiles* } & \multirow[b]{2}{*}{ Critical questions } \\
\hline Technique & Focus & Example & \\
\hline Modal profile & $\begin{array}{l}\text { The most } \\
\text { frequently } \\
\text { occurring } \\
\text { attributes }\end{array}$ & $\begin{array}{l}\text { When asked to state the preference for route of } \\
\text { administration: } 86 \%(69 / 80) \text { patients were comfortable } \\
\text { with an oral form (pills, drops or added to food), while } \\
15 \%(12 / 80) \text { chose smoking . } \\
\text { This was qualitised as: Most patients stated preference } \\
\text { for oral formulations, while a minority preferred inhaled } \\
\text { products. }\end{array}$ & \multirow{3}{*}{$\begin{array}{l}\text { What is this study trying to say about } \\
\text { patients' values? } \\
\text { Are patients' values and preferences } \\
\text { explicitly identified? If so, what are they? } \\
\text { How do participants' answers to the } \\
\text { questions provide insight into patients' } \\
\text { values and preferences, and their } \\
\text { influence on the choice of treatment for } \\
\text { chronic pain? } \\
\text { How different (or similar) are patients' } \\
\text { and carers' perspectives on medical } \\
\text { cannabis for chronic pain? } \\
\text { Are there other individual or contextual } \\
\text { factors (eg, age, gender, socioeconomic } \\
\text { status) that influence patients' values } \\
\text { and preferences towards medical } \\
\text { cannabis for chronic pain? }\end{array}$} \\
\hline Average profile & $\begin{array}{l}\text { Average of } \\
\text { the particular } \\
\text { variables }\end{array}$ & $\begin{array}{l}\text { Patients' concerns regarding medical cannabis using } \\
\text { a } 10 \text {-point scale }(0=\text { not concerned, } 10=\text { extremely } \\
\text { concerned) were, in order of important: side effects } \\
\text { (mean }=7.0 \pm 2.9) \text {, addiction }(6.6 \pm 3.2) \text {, tolerance }(6.2 \pm 3.2) \text {, } \\
\text { losing control or acting strangely }(6.2 \pm 3.3) \text {, and what } \\
\text { family and friends may think ( } 3.9 \pm 3.8) \text {. } \\
\text { This was qualitised as: Patients were generally most } \\
\text { concerned about the side effects of medical cannabis, } \\
\text { followed by addiction, tolerance, losing control or acting } \\
\text { strangely, and what family and friends may think. }\end{array}$ & \\
\hline $\begin{array}{l}\text { Comparative } \\
\text { profile }\end{array}$ & $\begin{array}{l}\text { A comparison } \\
\text { of key } \\
\text { outcomes }\end{array}$ & $\begin{array}{l}\text { Patients were asked to rate their values and concerns } \\
\text { regarding use of cannabis (strongly agree, agree, } \\
\text { disagree, strongly disagree and don't know). Significantly } \\
\text { more males, versus women, were concerned about } \\
\text { cannabis being addictive }(p=0.031) \text {, leading to the use } \\
\text { of more harmful substances }(p=0.036) \text {, and causing an } \\
\text { inability to think clearly ( } p=0.008) \text {. } \\
\text { This was qualitised as: Compared with females, } \\
\text { significantly more males were concerned about cannabis } \\
\text { being addictive, leading to the use of more harmful } \\
\text { substances, and causing an inability to think clearly. }\end{array}$ & \\
\hline
\end{tabular}

*We used the following criteria when 'qualitising' quantitative into qualitative data: Very few': reported by $10 \%$ or less of patients (if the sample was >100). 'Most common' and 'least common' were used when factors were reported in groups, to denote the factors that patients agreed with the most versus the least. The criteria above did not apply in these cases (eg, 'Recommendations from a medical professional was the least influential factor among patients when selecting cannabis.'). All or almost all': Reported by over $90 \%$ of patients; 'Most': Reported by $75 \%-90 \%$ of patients; 'Majority': Reported by $50 \%-75 \%$ of patients; 'Minority': Reported by $25 \%-50 \%$ of patients; 'Some': Reported by $10 \%-25 \%$ of patients; 'None or almost none': Reported by $10 \%$ or less of patients (if the sample was 100 or less).

narratives (table 1). ${ }^{12} 16$ By using inductive content analysis we synthesised the qualitised findings to produce review findings which addressed the key themes.

\section{Certainty of evidence}

For review findings from quantitative studies, we assessed the certainty of evidence according to the five GRADE domains (ie, risk of bias, imprecision, inconsistency, indirectness and small study effects). ${ }^{13} 1718$ For review findings from qualitative studies, we assessed the certainty of evidence according to the five GRADE Confidence in the Evidence from Reviews of Qualitative Research domains (ie, methodological limitations, relevance, coherence, adequacy and dissemination bias). ${ }^{19}$ We initially considered the certainty of evidence as high, and if serious or several minor or moderate concerns were detected in one or more domains, we rated down certainty of evidence by one or more levels to moderate, low or very low.

\section{Patient and public involvement}

We engaged three people living with chronic pain, one of whom used medical cannabis, to review our findings and advise if they were consistent with their experiences. Led by the MAGIC Evidence Ecosystem Foundation, a BMJ RapidRec panel of clinicians, methodologists and persons with lived experience of chronic pain were responsible for developing clinical practice recommendations for 
medical cannabis and chronic pain. Three patient partners were full members of the guideline panel and received training and support to optimise contributions throughout the guideline development process. The panel developed recommendations using the GRADE framework, available online through the MAGICapp, ${ }^{10}$ and considered evidence from systematic reviews on the effectiveness of medical cannabis, adverse events related to medical cannabis, opioid substitution with medical cannabis, and this review of patients' values and preferences regarding medical cannabis to manage chronic pain.

\section{RESULTS}

Our search retrieved 1838 records, of which 102 were deemed potentially eligible based on titles and abstracts. After full-text screening, 15 studies (reported in 16 articles) proved eligible for review, including nine quantitative studies, five qualitative studies and one mixedmethod study (figure 1, online supplemental appendices 5 and 6$)^{20-35}$

\section{Study characteristics}

Of the 15 studies, nine were conducted in the USA, two in the UK, two in Israel, one in Canada and one in Australia. Four studies were conducted between 2000 and 2009, and 11 were conducted between 2010 and 2019. The number of participants ranged from 34 to 1514 among quantitative studies, 18 to 150 in the qualitative studies, and 984 were enrolled in the mixed method study. All 15 studies included only chronic pain patients; no caregivers were enrolled (online supplemental appendix 5).

Among the nine quantitative and one mixed method studies, four were at serious and one at critical risk of bias due to lack of valid representation of the outcomes (eg,

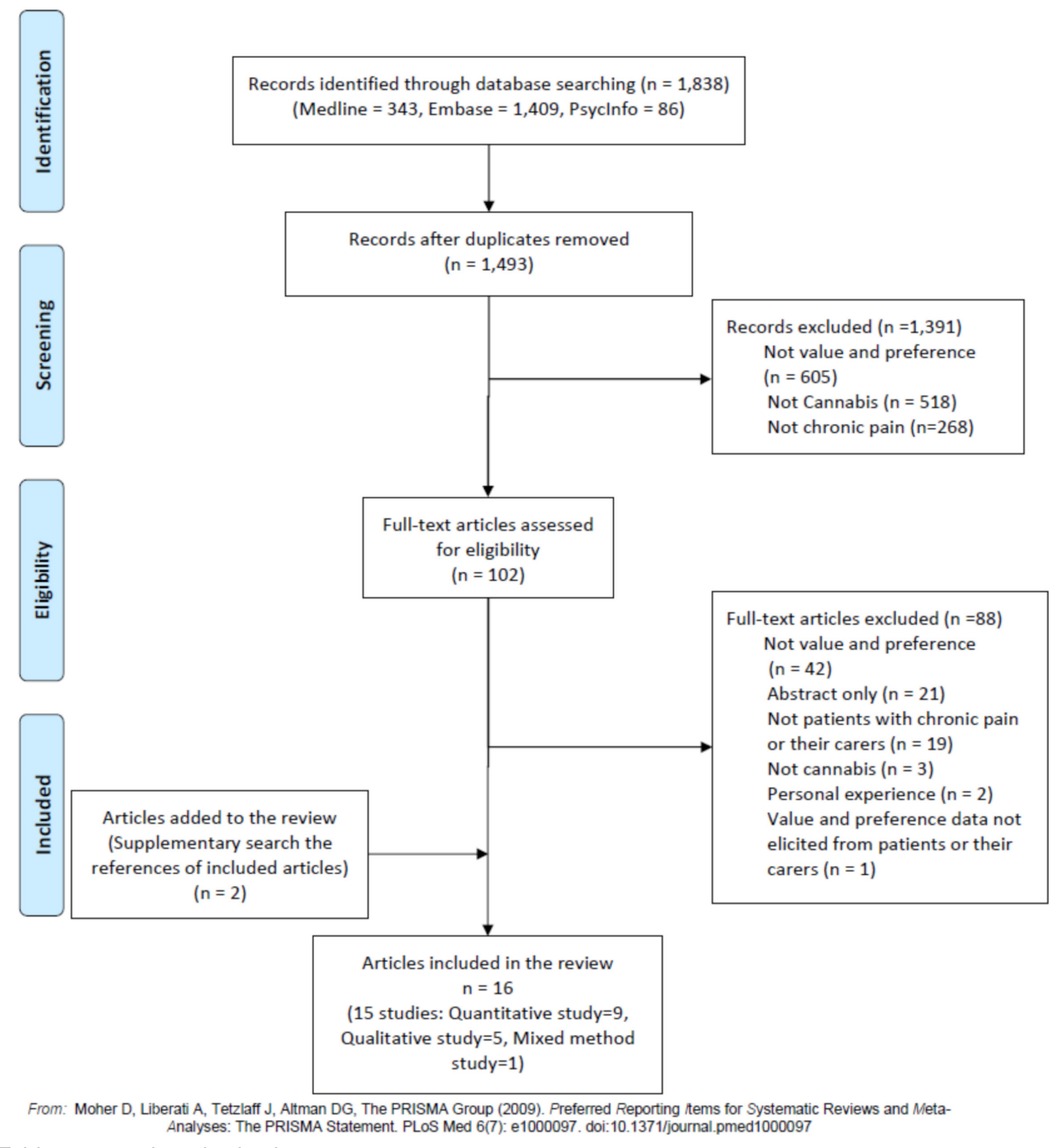

Figure 1 Evidence search and selection. 
beneficial or harmful outcomes of medical cannabis), low response rate (less than $80 \%$ ) and lack of reporting on how the authors confirmed participants' understanding of the measurement techniques (eg, questionnaire) (online supplemental appendix 7). Among the five qualitative studies, only one was at serious risk of bias due to inadequate research design and data collection, and lack of reporting on whether the relationship between researchers and participants had been adequately considered (online supplemental appendix 8).

\section{Findings}

We identified two key themes: values and preferences towards medical cannabis for chronic pain (seven quantitative studies (2185 participants)), three qualitative studies (95 participants) and one mixed-method study (984 participants)) and factors that influenced patient's decisions regarding use of medical cannabis (seven quantitative studies (4998 participants), five qualitative studies (263 participants) and one mixed-method study (984 participants)) (table 2, online supplemental appendix 9).

\section{Use of medical cannabis for chronic pain}

Low certainty evidence showed that patients had mixed levels of willingness to use medical cannabis and most patients who used medical cannabis reported positive attitudes toward its use. Most patients with advanced life-limiting illnesses were comfortable using cannabis for pain, ${ }^{25}$ while some other patients with chronic pain were unwilling or ambivalent about medical cannabis use. ${ }^{26}$ Non-white patients with advanced illness were more concerned about medical cannabis compared with white patients, but they remained comfortable using medical cannabis. ${ }^{25}$ People living with chronic pain who used medical cannabis believed it was effective for reducing their pain ${ }^{25} 273134$ and allowed them to reduce use of prescribed medications. ${ }^{27}$ Two qualitative studies found similar results. ${ }^{22} 28$

\section{Medical cannabis versus other pain medicines}

Patients with histories of substance use preferred medical cannabis over prescription opioids (low certainty). ${ }^{23}$ Some patients endorsed that medical cannabis was safer than other analgesics, and such beliefs were more prevalent among non-Christians and patients with colleges education or higher (very low certainty). ${ }^{25}$

\section{Different preparations of medical cannabis}

Moderate certainty evidence showed that most people living with chronic pain preferred using a blend of indica and sativa to manage their condition. ${ }^{21}$ There was no difference in the preference of cannabis strain between males and females, those who used cannabis for medical purposes only and those who endorsed medical and recreational use, or between novice and experienced users. ${ }^{21}$

Most patients preferred medical cannabis products with either balanced ratios of THC:CBD (37\%) or high CBD formulations $(46 \%)$ and only a minority (17\%) preferred high THC products (Moderate certainty). ${ }^{21} 33$
Specifically, women, novice users or those who endorsed use of cannabis for medical purposes only were more inclined to choose products with low THC and high CBD ratios, while males, those endorsing use of cannabis for both medical and recreational purposes, and experienced users preferred products with equal ratios of THC:CBD. ${ }^{21}$

Sex, reason for use, and experience with cannabis influenced preference towards route of administration (moderate certainty). ${ }^{21} 35$ Compared with male patients, women preferred to use tinctures and topical preparations as opposed to vaporising or smoking. ${ }^{21}$ Patients who used cannabis both recreationally and medically preferred smoking most, while those who used cannabis medically only preferred vaporising most. ${ }^{21}$ Experienced cannabis users endorsed multiple routes of administration compared with novice users who preferred vaporising. ${ }^{21}$ Most patients with advanced life-limiting illness preferred oral formulations (non-inhaled) of medical cannabis. ${ }^{25}$

\section{Factors influencing the decision to use medical cannabis}

High to moderate certainty evidence showed that most people living with chronic pain used medical cannabis for symptom relief. ${ }^{202} 232835$ Specifically, patients viewed medical cannabis as an effective approach to managing pain, ${ }^{20} 222335$ sleep, appetite and nausea. ${ }^{2035}$ Patients also reported that cannabis improved their emotional and mental well-being by reducing anxiety, depression and stress, ${ }^{20}{ }^{35}$ and increased their ability to focus and function. ${ }^{28}$ Most patients reported that cannabis facilitated a state of relaxation in which pain remained present but was easier to tolerate. ${ }^{28}$

Moderate certainty evidence showed that factors related to patients' unwillingness to use medical cannabis include major side effects (eg, losing control or acting strangely), ${ }^{20} 232627313435$ addiction or tolerance, 2627313435 and negative social consequences (eg, stigma). ${ }^{2025} 2631323435$ Older age was associated with greater hesitancy to use medical cannabis, as was concerns about negative opinions from others which might lead to relationship problems or disagreements with loved ones. ${ }^{25} 263134$ Some patients reported that stigma affected their comfort in asking healthcare providers about cannabis as a treatment option, and their willingness to use medical cannabis in a public setting. ${ }^{32}$ Moderate certainty evidence showed that cost, legal status, and accessibility of medical cannabis also influenced use. ${ }^{2023-25313435}$

Factors influencing the choice of different preparations of medical cannabis

Low certainty evidence suggested that most patients chose medical cannabis products based on cannabinoid content (ie, THC or CBD potency, ratio of THC and CBD), recommendations from dispensary employees, described effects (eg, pain relief), strain of cannabis plant (ie, sativa, indica, hybrid), smell or varietal name. ${ }^{21-232830}$ A higher proportion of males selected cannabis products based on cannabinoid content, cannabis variety, visual properties and smell, while a higher proportion 
Table 2 Review findings and certainty of evidence

Review findings*

Type of research

evidence: Reference no Certainty of evidence

Values and preferences towards medical cannabis for chronic pain

Use of medical cannabis for chronic pain

Chronic pain patients had mixed levels of comfort or

willingness to use medical cannabis.

Quantitative: 25, 26, 27 Low: Risk of bias and indirectness

Qualitative: 22

Low: Minor concerns about relevance, serious adequacy concerns

Most patients who use medical cannabis had a positive

Quantitative: 25, 27, 29, Low: Risk of bias and indirectness

attitude towards its use for pain relief.

31,34

Qualitative: 28

Moderate: Serious adequacy concerns

Medical cannabis over other pain medicines

Patients with chronic pain and substance use histories Qualitative: 23

preferred medical cannabis over prescription opioids.

Qualitative: $23 \quad$ Low: Moderate methodological limitations and moderate adequacy concerns

$\begin{array}{ll}\begin{array}{l}\text { Some patients believed that medical cannabis is safer than } \\ \text { morphine and other strong pain killers. }\end{array} & \begin{array}{l}\text { Quantitative: } 25 \\ \text { imprecision }\end{array}\end{array}$

\section{Different preparations of medical cannabis}

Cannabis variety (ie, sativa, indica, hybrid)

Most patients preferred medical cannabis with a blend of $\quad$ Quantitative: $21 \quad$ Moderate: Risk of bias

indica and sativa, regardless of gender, reasons for use, and

cannabis experience level.

\section{Cannabis content (ie, THC or CBD potency, ratio of THC and CBD)}
A balanced ratio of THC:CBD was the most preferred
Quantitative: 21, 33
Moderate: Risk of bias

preparation, but gender, reason for use, and cannabis

experience level influenced patients' preference for cannabis

ratio.

\section{Cannabis administration route}

Gender, reason for use and cannabis experience level influenced patients' preferred cannabis administration

Quantitative: 21

Mixed method: 35

Moderate: Risk of bias

routes.

Most patients with advanced life-limiting illness preferred an Quantitative: $25 \quad$ Low: Risk of bias and imprecision oral form (non-inhaled) of medical cannabis.

\section{Factors that influenced patient's decision regarding use of medical cannabis}

\section{Factors influenced the choice of medical cannabis use}

Most patients used medical cannabis because it improved symptoms associated with pain, mental health and other medical conditions.

Most patients were motivated to use medical cannabis to reduce use of prescription medication.

Qualitative: 20, 22, 23, 28 High

Mixed method: $35 \quad$ Moderate: Risk of bias

Quantitative study: 27

Moderate: Risk of bias

Qualitative study: 22

Moderate: Moderate adequacy concerns

The majority of patients expressed that their cannabis use was influenced by positive social consequences, such as

Quantitative: 25, 31,34

Moderate: Risk of bias social support from friends and family.

Most patients expressed concerns with using medical cannabis, and described a range of adverse effects.
Quantitative: 26, 27, 31, 34 Moderate: Risk of bias

Mixed method: 35

Qualitative : 20, 23

Moderate: Moderate methodological concerns

Most patients expressed that their cannabis use was influenced by negative social consequences, such as stigma. Mixed method: 35

Quantitative: 25, 26, 31, 4 Moderate: Risk of bias

Qualitative: 20, 23
Qualitative: 20, 32

Quantitative: 24, 25, 31, 34 Moderate: Risk of bias Mixed method: 35

Moderate: Moderate methodological limitations

The cost, legal status and accessibility of medical cannabis influenced patients' decisions to use medical cannabis.
Moderate: Moderate methodological limitations 
Factors influenced the choice of different preparations of medical cannabis

$\begin{array}{lll}\begin{array}{l}\text { Patients chose medical cannabis products mainly based on } \\ \text { cannabinoid content, recommendations from dispensary } \\ \text { employees, described effects and side effects, strain of } \\ \text { cannabis plant, smell and flower appearance. }\end{array} & \begin{array}{l}\text { Quantitative: 21, } 30 \\ \text { Qualitative: 22, 23, 28 }\end{array} & \begin{array}{l}\text { Low: Risk of bias and indirectness } \\ \text { and serious adequacy concerns }\end{array} \\ \begin{array}{l}\text { Gender, reason for use, and level of use experience were } \\ \text { factors influencing patients' selection of cannabis products. }\end{array} & \text { Quantitative: 21 } & \text { Moderate: Risk of bias }\end{array}$

*We used the following criteria when 'qualitising' quantitative into qualitative data: 'Very few': Reported by $10 \%$ or less of patients (if the sample was 101 or more). 'Most common' and 'least common' were used when factors were reported in groups, to denote the factors that patients agreed with the most versus the least. The criteria above did not apply in these cases (eg, 'Recommendations from a medical professional was the least influential factor among patients when selecting cannabis'). 'All or almost all': Reported by over $90 \%$ of patients; 'Most': Reported by $75 \%-90 \%$ of patients; 'Majority': Reported by $50 \%-75 \%$ of patients; 'Minority': Reported by $25 \%-50 \%$ of patients; 'Some': Reported by $10 \%-25 \%$ of patients; 'None or almost none': Reported by $10 \%$ or less of patients (if the sample was 100 or less).

CBD, cannabidiol; THC, delta-9-tetrahydrocannabinol.

of females consulted with a medical professional when choosing cannabis products (moderate certainty). ${ }^{21}$

Patients who used cannabis both medically and recreationally were more likely to select cannabis products based on cannabinoid content, cannabis variety, described effects, visual properties, smell, recommendations from friends and the product name, while those who only used cannabis medically were more likely to prioritise recommendations from dispensary employees or medical professionals (moderate certainty). ${ }^{21}$

\section{DISCUSSION}

Values and preferences among patients with chronic pain towards the use of medical cannabis are highly variable. Improvement of symptoms and reduction of prescription medications are important factors that positively influence patients' decision to use medical cannabis, while concerns about addiction, losing control, acting strangely and negative social consequences are associated with unwillingness to use medical cannabis. Cost, legal status and accessibility are also important factors. Patients who endorsed use of cannabis for only medical reasons preferred high CBD or similar ratios of THC:CBD products, whereas those endorsing use of both medical and recreational purposes were more likely to use high THC products. Further, patients with chronic pain endorsing both medical and recreational use were more likely to prefer smoking cannabis, versus patients who endorsed only medical use who preferred vaporising. Our findings were consistent across bodies of evidence (quantitative, qualitative and mixed-method studies). The certainty of evidence for most findings was moderate, predominantly due to risk of bias or imprecision/adequacy.

We asked three patient partners on the BMJ rapid recommendation panel for their comments on the findings of this systematic review. In particular, (1) whether our findings reflected their experiences, and (2) if some of the findings were different from their experience, what were possible reasons? The patient partners agreed that all except one of our review findings (table 2) reflected their experiences with cannabis. Specifically, they suggested that patients who are using medical cannabis may not receive support from family or friends due to stigma and misinformation about cannabis use.

Our findings that some patients select medical cannabis based on properties that dispensers attributed to strain type (indica or sativa), represents an opportunity for education. When these strains were originally characterised, sativa was shown to produce higher amounts of CBD whereas indica strains of cannabis produced high levels of THC. At present, however, commercially available cannabis plants and products have been extensively interbred to produce a multitude of unique strains. ${ }^{36}$ As such, the only reliable way to determine the composition of any form of medical cannabis is through accurate reporting of the cannabinoid (eg, THC, CBD) content.

We found important differences between patients who use cannabis for medical reasons only and those who report combined use (medical and recreational) in preferences regarding cannabis content and route of administration. Observational studies have shown that most consumers of cannabis endorse medical and recreational use, ${ }^{3738}$ which presents a challenge to therapeutic use. Recreational users often prioritise cannabis with high THC concentrations, a psychotropic cannabinoid that is associated with greater harms than CBD. ${ }^{39} 40$ Patients who use cannabis for both medical and recreational purposes are also more likely to prefer inhaled forms of administration, which has a much faster onset and greater bioavailability than ingestion but also entails pulmonary risk factors due to inhalation of toxins and particulate matter. ${ }^{41}$ Therapeutic use of cannabis should prioritise formulations supported by evidence, administered in a manner that prioritises both safety and effectiveness. 


\section{Strengths and limitations of the review}

Strengths of this review include explicit eligibility criteria, an extensive search strategy, and duplicate assessment of eligibility and risk of bias. The use of complementary bodies of evidence (qualitative, quantitative and mixedmethods) and the use of the GRADE approach to assess the certainty of evidence allowed greater confidence in the interpretation of results.

This study also had limitations. Most of the eligible studies (13 out of 15 studies) are from high-income countries, reflecting values and preferences of patients living in better healthcare service systems with health insurance coverage. The generalisability of our findings to other populations in uncertain. In addition, we synthesised and reported patients' willingness to use medical cannabis despite the limitation that most studies did not provide participants with sufficient information about the benefits and harms of medical cannabis. Studies failed to consistently report participants' socioeconomic status, educational level and religious beliefs, limiting exploration of the effect of these characteristics on values and preferences.

\section{Implications}

Our findings have direct implications for clinicians attending people living with chronic pain who are considering use of medical cannabis. Benefits (effect on pain and reduction of prescription medications), harms (adverse effects), burdens (negative social consequences, cost) and accessibility (including legal status) of medical cannabis all appear to influence patients' decisions related to use. However, we did not identify any studies that considered how patients prioritised these factors. Subsequent research should address this issue. In addition, how patient characteristics (eg, medical conditions, social economic status, religious beliefs) affect their values and preferences is another issue worth addressing in subsequent research.

\section{CONCLUSIONS}

There exists high variability of values and preferences towards medical cannabis among people living with chronic pain, particularly related to their willingness to use medical cannabis. These findings suggest that an individualised patient-centred approach, such as shared decision making, should be emphasised for empowering patients to make choices that best suit their own values and preferences and accommodate their context.

\footnotetext{
Author affiliations

${ }^{1}$ Pharmacy Department/Evidence-based Pharmacy Center, West China Second University Hospital, Chengdu, Sichuan, China

${ }^{2}$ Department of Health Research Methods, Evidence \& Impact, McMaster University, Hamilton, Ontario, Canada

${ }^{3}$ Michael G. DeGroote Institute for Pain Research and Care, McMaster University, Hamilton, Ontario, Canada

${ }^{4}$ Institute for Health Policy, Management and Evaluation, University of Toronto, Toronto, Ontario, Canada
}

${ }^{5}$ Women's College Institute for Health System Solutions and Virtual Care, Women's College Hospital, Toronto, Ontario, Canada

${ }^{6}$ Ferkauf Graduate School of Psychology, Yeshiva University, New York, New York, USA

${ }^{7}$ Department of Medicine, Lovisenberg Diaconal Hospital, Oslo, Norway

${ }^{8}$ Division of General Internal Medicine \& Division of Clinical Epidemiology, University Hospitals of Geneva, Geneva, Switzerland

${ }^{9}$ The Michael G. DeGroote Centre for Medicinal Cannabis Research, McMaster University, Hamilton, Ontario, Canada

${ }^{10}$ Department of Anesthesia, McMaster University, Hamilton, Ontario, Canada

${ }^{11}$ The Michael G. DeGroote National Pain Centre, McMaster University, Hamilton, Ontario, Canada

${ }^{12}$ Chronic Pain Centre of Excellence for Canadian Veterans, Hamilton, Ontario, Canada

Twitter Thomas Agoritsas @ThomasAgoritsas and Jason W Busse @JasonWBusse

Acknowledgements We thank Rachel Couban (McMaster University) for performing the literature search; we thank Ray Zhang (McMaster University) for providing consult with the certainty of evidence rating; we thank Shelly-Anne Li (University of Toronto) for providing suggestions on data synthesis; we thank our patient partners for providing comments on review findings.

Contributors LZ, XW, NK, SA, YS and ME identified and selected the studies. LZ, XW, NK, SA, YS and MAE collected the data. LZ, LL, XW, NK and SA analysed the data and assessed the certainty of the evidence. AFH, TA, GHG and JWB provided advice at different stages. LZ, LL, XW, NK and SA drafted the manuscript. All authors revised the manuscript and approved the final version of the manuscript.

Funding XW is supported by a postdoctoral fellowship from the Michael G. DeGroote Institute for Pain Research and Care.

Competing interests None declared.

Patient consent for publication Not required.

Provenance and peer review Not commissioned; externally peer reviewed.

Data availability statement Data are available on reasonable request from the corresponding author.

Supplemental material This content has been supplied by the author(s). It has not been vetted by BMJ Publishing Group Limited (BMJ) and may not have been peer-reviewed. Any opinions or recommendations discussed are solely those of the author(s) and are not endorsed by BMJ. BMJ disclaims all liability and responsibility arising from any reliance placed on the content. Where the content includes any translated material, BMJ does not warrant the accuracy and reliability of the translations (including but not limited to local regulations, clinical guidelines, terminology, drug names and drug dosages), and is not responsible for any error and/or omissions arising from translation and adaptation or otherwise.

Open access This is an open access article distributed in accordance with the Creative Commons Attribution Non Commercial (CC BY-NC 4.0) license, which permits others to distribute, remix, adapt, build upon this work non-commercially, and license their derivative works on different terms, provided the original work is properly cited, appropriate credit is given, any changes made indicated, and the use is non-commercial. See: http://creativecommons.org/licenses/by-nc/4.0/.

\section{ORCID iDs}

Linan Zeng http://orcid.org/0000-0001-9892-2000

Natasha Kithulegoda http://orcid.org/0000-0001-6207-9699

Thomas Agoritsas http://orcid.org/0000-0002-6182-9969

Jason W Busse http://orcid.org/0000-0002-0178-8712

\section{REFERENCES}

1 James SL, Abate D, Abate KH, et al. Global, regional, and national incidence, prevalence, and years lived with disability for 354 diseases and injuries for 195 countries and territories, 1990-2017: a systematic analysis for the global burden of disease study 2017. The Lancet 2018;392:1789-858.

2 Goldberg DS, McGee SJ. Pain as a global public health priority. BMC Public Health 2011;11:1-5.

3 Jackson T, Thomas S, Stabile V, et al. Prevalence of chronic pain in low-income and middle-income countries: a systematic review and meta-analysis. Lancet 2015;385 Suppl 2:S10. 
4 Hill KP. Medical marijuana for treatment of chronic pain and other medical and psychiatric problems: a clinical review. JAMA 2015;313:2474-83.

5 Medical cannabis: bridging the evidence gap. The Lancet Rheumatology 2019;4:e195.

6 Hill KP. Medical marijuana: more questions than answers. J Psychiatr Pract 2014:20:389.

7 The National Institute for health and care excellence (NICE) cannabisbased medicinal products (NG144), 2019. Available: https://www. nice.org.uk/guidance/ng144/chapter/Recommendations\#chronicpain [Accessed 7 Mar 2021].

8 Yadav V, Bever C, Bowen J, et al. Summary of evidence-based guideline: complementary and alternative medicine in multiple sclerosis: report of the Guideline Development Subcommittee of the American Academy of Neurology. Neurology 2014;82:1083-92.

9 Guyatt GH, Haynes RB, Jaeschke RZ, et al. Users' guides to the medical literature: XXV. evidence-based medicine: principles for applying the users' guides to patient care. evidence-based medicine Working group. JAMA 2000;284:1290-6.

10 Busse JW VP, Zeng L, et al. Medical cannabis for chronic pain: a clinical practice guideline. BMJ.

11 Selva A, Solà I, Zhang Y, et al. Development and use of a content search strategy for retrieving studies on patients' views and preferences. Health Qual Life Outcomes 2017;15:126.

12 Valli C, Rabassa M, Johnston BC, et al. Health-Related values and preferences regarding meat consumption: a mixed-methods systematic review. Ann Intern Med 2019;171:742-55.

13 Zhang Y, Alonso-Coello P, Guyatt GH, et al. GRADE guidelines: 19. assessing the certainty of evidence in the importance of outcomes or values and preferences-Risk of bias and indirectness. J Clin Epidemiol 2019;111:94-104.

14 Critical Appraisal Skills Programme. CASP qualitative research checklist; 2018.

15 Barnett-Page E, Thomas J. Methods for the synthesis of qualitative research: a critical review. BMC Med Res Methodol 2009;9:1-11.

16 Karimi M, Clark AM. How do patients' values influence heart failure self-care decision-making?: a mixed-methods systematic review. Int J Nurs Stud 2016;59:89-104.

17 Zhang Y, Coello PA, Guyatt GH, et al. GRADE guidelines: 20. assessing the certainty of evidence in the importance of outcomes or values and preferences-inconsistency, imprecision, and other domains. J Clin Epidemiol 2019;111:83-93.

18 Guyatt GH, Oxman AD, Schünemann HJ, et al. GRADE guidelines: a new series of articles in the Journal of clinical epidemiology. J Clin Epidemiol 2011;64:380-2.

19 Lewin S, Bohren M, Rashidian A, et al. Applying GRADE-CERQual to qualitative evidence synthesis findings-paper 2: how to make an overall CERQual assessment of confidence and create a summary of qualitative findings table. Implement Sci 2018;13:10.

20 Bigand T, Anderson CL, Roberts ML, et al. Benefits and adverse effects of cannabis use among adults with persistent pain. Nurs Outlook 2019;67:223-31.

21 Boehnke KF, Scott JR, Litinas E, et al. Cannabis use preferences and decision-making among a cross-sectional cohort of medical cannabis patients with chronic pain. J Pain 2019;20:1362-72.

22 Bruce D, Brady JP, Foster E, et al. Preferences for medical marijuana over prescription medications among persons living with chronic conditions: alternative, complementary, and tapering uses. J Altern Complement Med 2018;24:146-53.
23 Cooke AC, Knight KR, Miaskowski C. Patients' and clinicians' perspectives of co-use of cannabis and opioids for chronic non-cancer pain management in primary care. Int J Drug Policy 2019;63:23-8.

24 Degenhardt L, Lintzeris N, Campbell G, et al. Experience of adjunctive cannabis use for chronic non-cancer pain: findings from the pain and opioids in treatment (point) study. Drug Alcohol Depend 2015;147:144-50.

25 Gallagher R, Best JA, Fyles G, et al. Attitudes and beliefs about the use of cannabis for symptom control in a palliative population. Journal of Cannabis Therapeutics 2003;3:41-50.

26 Gill A, Williams AC, ACdC W. Preliminary study of chronic pain patients' concerns about cannabinoids as analgesics. Clin J Pain 2001;17:245-8.

27 Heng M, McTague MF, Lucas RC, et al. Patient perceptions of the use of medical marijuana in the treatment of pain after musculoskeletal trauma: a survey of patients at 2 trauma centers in Massachusetts. J Orthop Trauma 2018;32:e25-30.

28 Lavie-Ajayi M, Shvartzman P. Restored self: a phenomenological study of pain relief by cannabis. Pain Med 2019;20:2086-93.

29 Rochford C, Edgeworth D, Hashim M, et al. Attitudes of Irish patients with chronic pain towards medicinal cannabis. Ir J Med Sci 2019;188:267-72.

30 Sexton M, Cuttler C, Finnell JS, et al. A cross-sectional survey of medical cannabis users: patterns of use and perceived efficacy. Cannabis Cannabinoid Res 2016;1:131-8.

31 Zarrabi AJ, Welsh JW, Sniecinski R, et al. Perception of benefits and harms of medical cannabis among seriously ill patients in an outpatient palliative care practice. J Palliat Med 2020;23:558-62.

32 Satterlund TD, Lee JP, Moore RS. Stigma among California's medical marijuana patients. J Psychoactive Drugs 2015;47:10-17.

33 Notcutt W, Price M, Miller R, et al. Initial experiences with medicinal extracts of cannabis for chronic pain: results from 34 ' $\mathrm{N}$ of 1 ' studies. Anaesthesia 2004;59:440-52.

34 Singh V, Zarrabi AJ, Curseen KA, et al. Concerns of patients with cancer on accessing cannabis products in a state with restrictive medical marijuana laws: a survey study. $J$ Oncol Pract 2019;15:531-8.

35 Piper BJ, Beals ML, Abess AT, et al. Chronic pain patients perspectives of medical cannabis. Pain 2017;158:1373-9.

36 Piomelli D, Russo EB. The Cannabis sativa Versus Cannabis indica Debate: An Interview with Ethan Russo, MD. Cannabis Cannabinoid Res 2016;1:44-6.

37 Turna J, Balodis I, Munn C, et al. Overlapping patterns of recreational and medical cannabis use in a large community sample of cannabis users. Compr Psychiatry 2020;102:152188.

38 Pacula RL, Jacobson M, Maksabedian EJ. In the weeds: a baseline view of cannabis use among legalizing states and their neighbours. Addiction 2016;111:973-80.

39 Expert Committee on Drug Dependence Fortieth Meeting Cannabidiol (CBD) critical review report. Geneva; 2018.

40 Hall W. What has research over the past two decades revealed about the adverse health effects of recreational cannabis use? Addiction 2015:110:19-35.

41 National Institute on Drug Abuse. Marijuana research report, 2020. Available: https://www.drugabuse.gov/publications/research-reports/ marijuana/what-are-marijuanas-effects-lung-health 